\title{
Design and Study of Online Fuzzy Risk Score Analyzer for Diabetes Mellitus
}

\author{
${ }^{1}$ SunithaKarnam Anantha, ${ }^{2}$ Senthil Kumar Natarajan and ${ }^{1}$ Subransu Sekar Dash \\ ${ }^{1}$ School of Electrical and Electronics Engineering, \\ Faculty of Engineering and Technology, SRM University, Chennai, India \\ ${ }^{2}$ Department of Electrical and Electronics Engineering, Mepco Schlenk Engineering College, Sivakasi, India
}

Received 2013-07-26, Revised 2013-08-13; Accepted 2013-08-22

\begin{abstract}
The aim of this study is to determine the risks of various subjects to type 2 Diabetes and its dependence on the different subject records. A Fuzzy based system was designed to find the risk scores for diabetes based on risk score derived from Chennai Urban Rural Epidemiology Study (CURES). The risk score that has been adapted into the system is referred to as Indian Diabetes Risk Score (IDRS). The variables employed in it are age, gender, waist, exercise and history of diabetes. A database of subject records was collected from hundred random individuals from southern regions of India. A comparative study was performed on these records between the normal and fuzzified risk score based on IDRS. The program has been designed using Lab VIEW with Fuzzy System Designer being used for fuzzy rule execution. The details are transmitted online through web page to the physicians who can provide assistance in prevention of diabetes. The obtained risk scores of the subjects are used to improve the lifestyle and delay the onset of diabetes to the maximum possible. This system can be implemented in rural regions where experienced medical assistance may not be available. This system would form an ideal part of the current developments in medicine where physical physician presence is not required due to the buttress provided by advancements in computer technology. The aim of this study is to determine the risks of various subjects to type 2 Diabetes and its dependence on the different subject records. A Fuzzy based system was designed to find the risk scores for diabetes based on risk score derived from Chennai Urban Rural Epidemiology Study (CURES). The risk score that has been adapted into the system is referred to as Indian Diabetes Risk Score (IDRS). The variables employed in it are age, gender, waist, exercise and history of diabetes. A database of subject records was collected from hundred random individuals from southern regions of India. A comparative study was performed on these records between the normal and fuzzified risk score based on IDRS. The program has been designed using Lab VIEW with Fuzzy System Designer being used for fuzzy rule execution. The details are transmitted online through web page to the physicians who can provide assistance in prevention of diabetes. The obtained risk scores of the subjects are used to improve the lifestyle and delay the onset of diabetes to the maximum possible. This system can be implemented in rural regions where experienced medical assistance may not be available. This system would form an ideal part of the current developments in medicine where physical physician presence is not required due to the buttress provided by advancements in computer technology.
\end{abstract}

Keywords: Chennai Urban Rural Epidemiology Study-CURES, Fuzzy System Analyzer, IDRS, Indian Diabetes Risk Score-IDRS, Lab VIEW, Type 2 Diabetics, Risk Score

\section{INTRODUCTION}

The incidence of diabetes is on the rise and it is accelerating due to various factors related to lifestyle changes (Knowler et al., 2002). Prevention of diabetes is extremely important as the disease is spreading like an epidemic. Though there is increased awareness about diabetes there are many undetected cases of

Corresponding Author: SunithaKarnam Anantha, School of Electrical and Electronics Engineering, Faculty of Engineering and Technology, SRM University, Chennai, India 
diabetes. Hence there is a necessity for systems that can help to detect pre-diabetic stages (Lindstrom and Tuomilehto, 2003; Wild et al., 2004). This will facilitate individuals to improve their lifestyle through exercise and diet. These kind of precautions will cause to delay the onset of diabetes and in some cases prevent it too (Aekplakorn et al., 2006; Schulze et al., 2007; Mohan et al., 2005). This study deals with the design of such a system that will calculate the diabetes risk score and help in early detection and prevention of type 2 diabetes.

\section{BACKGROUND}

\subsection{Awareness}

India has been declared to be the diabetic capital of the world with over 5 crores diabetic patients. The number of patients is increasing continuously and steps need to be taken to curb the rise. The first step towards tackling the disease is knowledge of the disease symptoms and treatment. Awareness about diabetes is low in educated population and almost nil in the rural areas. Therefore there is immense potential for ways to bring awareness in general public. This can be achieved by allowing them to take part in questionnaires based on risk scores.

These results would enable them to comprehend their medical conditions. Also it will educate them about how to prevent or fight the disease in future. Few individuals were selected for case study and asked specific questions. An individual was found to be suffering from diabetes and taking medications. But still that person had tested the blood glucose levels only two times for the past two years. This shows that even diabetic patients are in the dark about the steps to be taken to reduce the complications arising due to diabetes.

\subsection{Indian Diabetes Risk Score}

IDRS uses four risk factors of age, abdominal obesity, family history of diabetes mellitus and physical activity to determine risk score. Beta coefficient were derived based on a multiple logistic regression analysis using undiagnosed diabetes mellitus as the dependent variable. The beta coefficients were modified so as to obtain a maximum possible score of hundred. The IDRS thus generated has been selected for finding the risk of people in India. The score is generally calculated by giving specific score for each factor. The age factor adds 0 for less than 35, 20 for range 35-49 and 30 for more than 50 years.

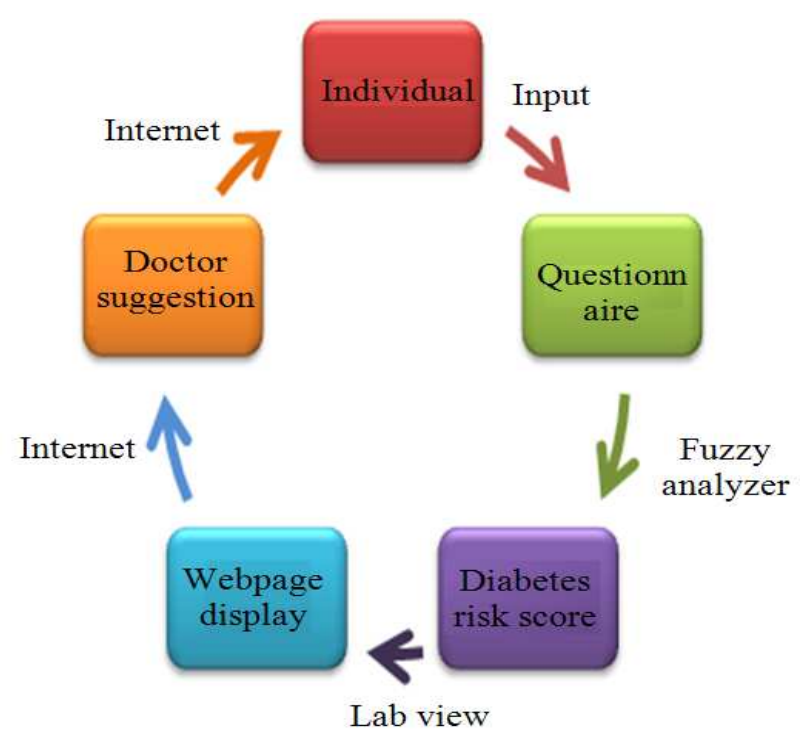

Fig. 1. Flow of program

The waist factor has different range for males and females; adds 0 for less than 80 , less than 90 and 10 for range $80-89,90-99,20$ for more than 90, more than 100 for females and males respectively. Both exercise and strenuous work add 0 , either one of thae two adds 20 and absence of both adds 30 to the score. No family history adds 0 , for either parent adds 10 and for both parents 20 .

\subsection{Design and Implementation of Online System}

The interactive system physically consists of two computers connected to each other by internet protocol. The program developed is running on both ends of the system. One end is the patient or individual and the other end is the physician. The program includes a display where the individual can enter the details using which the fuzzified IDRS is calculated. The program has been coded in Lab VIEW and hence the user interface and logic behind the program are developed simultaneously. A fuzzy based risk score has been developed for risk score calculation using this risk score which increases its accuracy. The fuzzy controller is more accurate than the normal controller because instead of being either true or false, a partial true case can also be declared. The patient may pose questions to the physician and get suggestions about lifestyle changes and treatment of diabetes. The flow of the program is shown in Fig. 1. 


\section{FUZZY SYSTEM}

The fuzzy system is implemented using fuzzy system designer in Lab VIEW. The design procedure includes two basic steps i.e., formation of membership functions for each linguistic variable and formation of rules. The Procedure is illustrated in Fig. 2.

Age are age 0 to 35,25 to 55 and 45 to 100 , ranging from 0 to 100. The membership functions of waist are 0 to 82,70 to 105 and 90 to 140 ranging for females and 0 to 92 , 80 to 115 and 100 to 150 for males. The risk has eleven

Membership functions ranging from 0 to 100 . The membership functions of these variables are shown in Fig. 3-5.

The rules are formed using if then statements where a certain combination of each of the inputs' membership functions give a particular risk score. The Number of rules depends upon the number of input variables and the total of membership functions for each input variable. All different possible combinations need to be added to the rules so as to obtain the exact score calculation for the mid values. The total number of rules built for IDRS is 162 . The rules creation window is shown in Fig. 6.

These systems may include simple questionnaires for disease prevention by pre-diagnosis, detection and treatment. They require basic equipments to be set up at the test sites. The information needs to be collected in an intelligent manner where the questions to be answered by the patients are simple enough but provide deep insights into the analysis of the disease. The system should be made to be user friendly and require no prior computer knowledge so that it is Comprehensible even to the illiterate people. The implementation of such systems is required not in places with established hospitals and developed areas but where availability of life essentials is limited. The rural areas of India lack basic medical facilities and not many educated individuals want their life to be restricted to the villages to serve the public. Thus the online systems provide for the physicians to be able to interact with their patients without being physically present online.

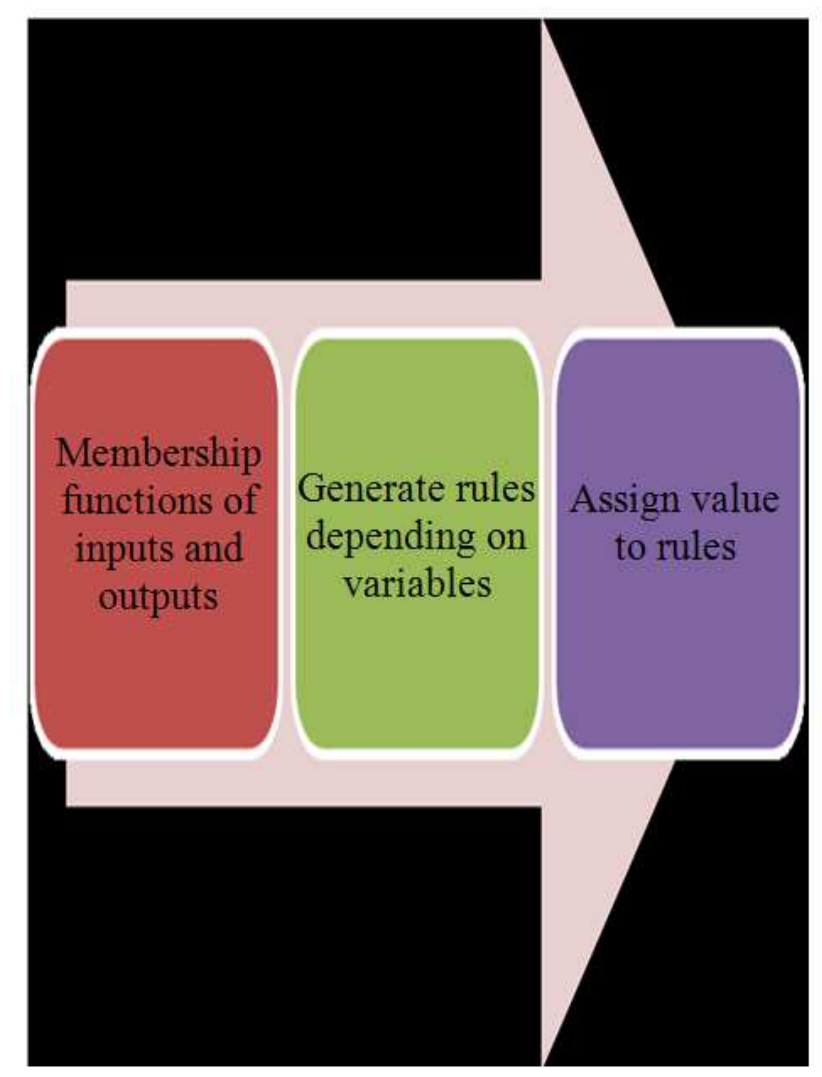

Fig. 2. Fuzzy system flow 
SunithaKarnam Anantha et al. / American Journal of Applied Sciences 10 (9): 1124-1133, 2013

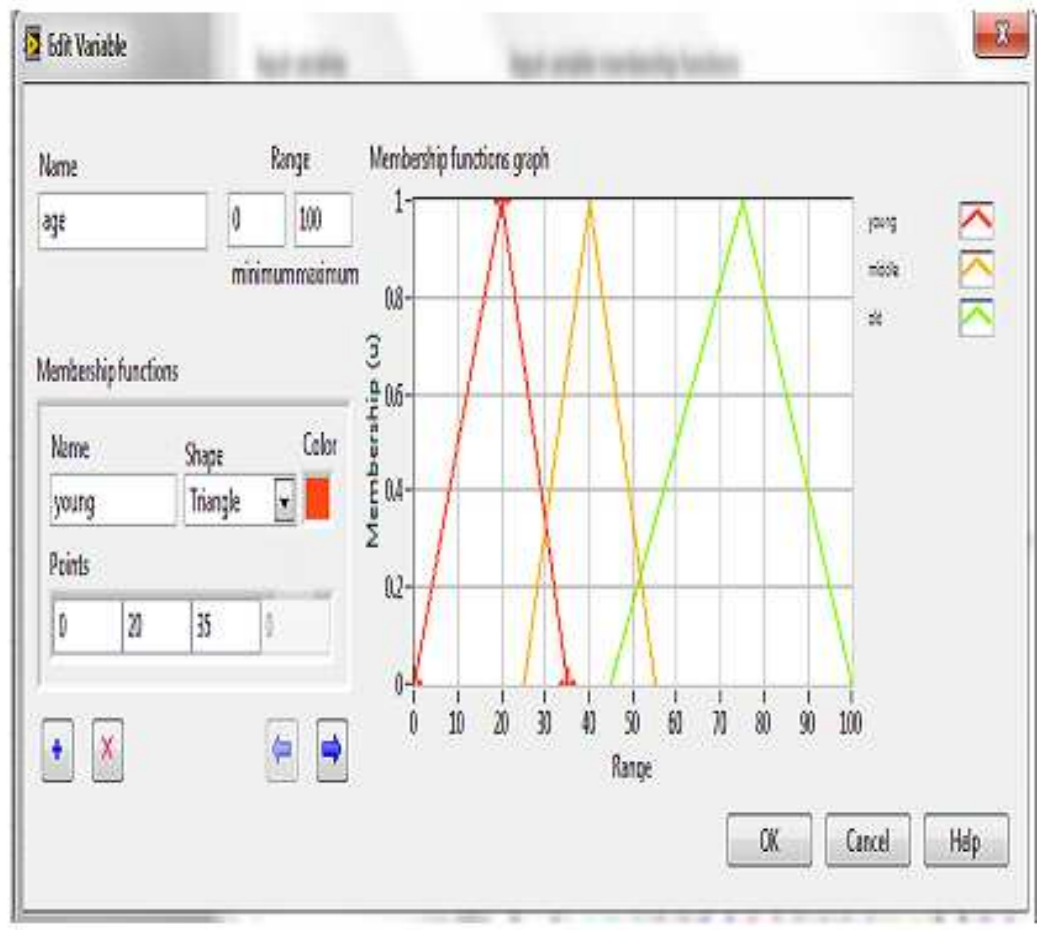

Fig. 3. Age membership functions

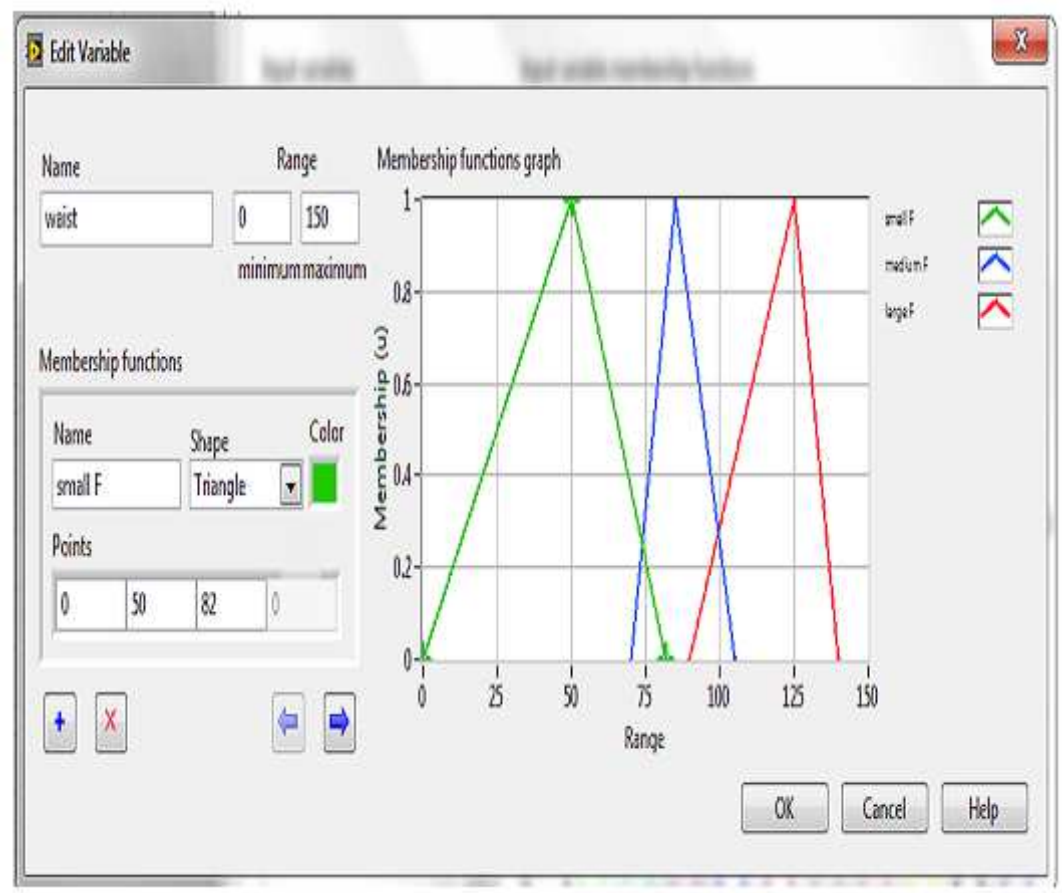

Fig. 4. Waist membership functions 
SunithaKarnam Anantha et al. / American Journal of Applied Sciences 10 (9): 1124-1133, 2013

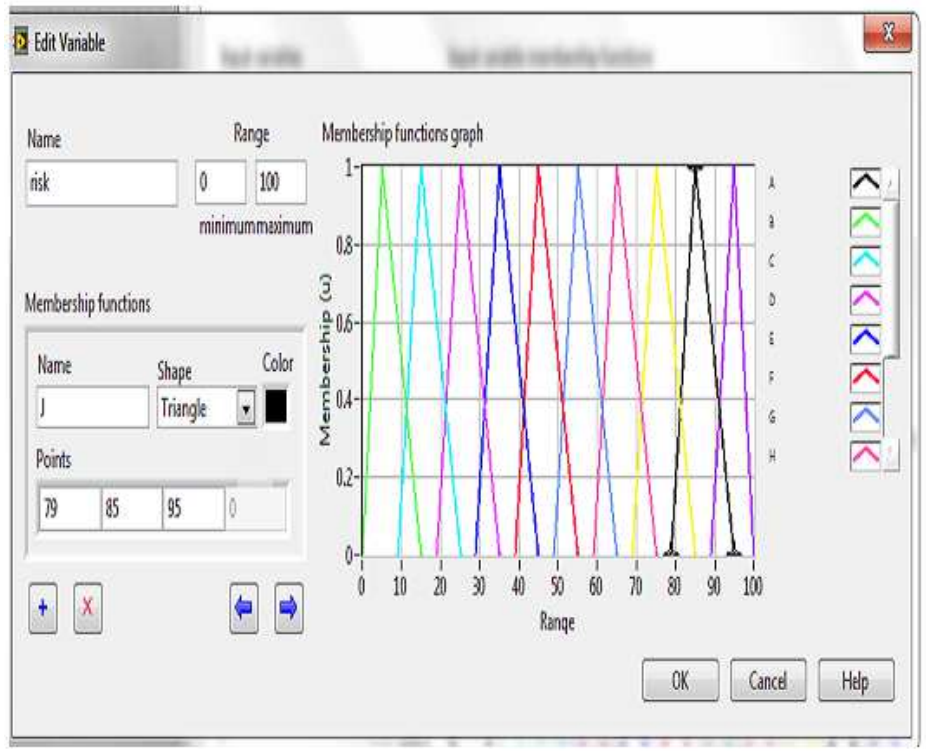

Fig. 5. Risk membership functions

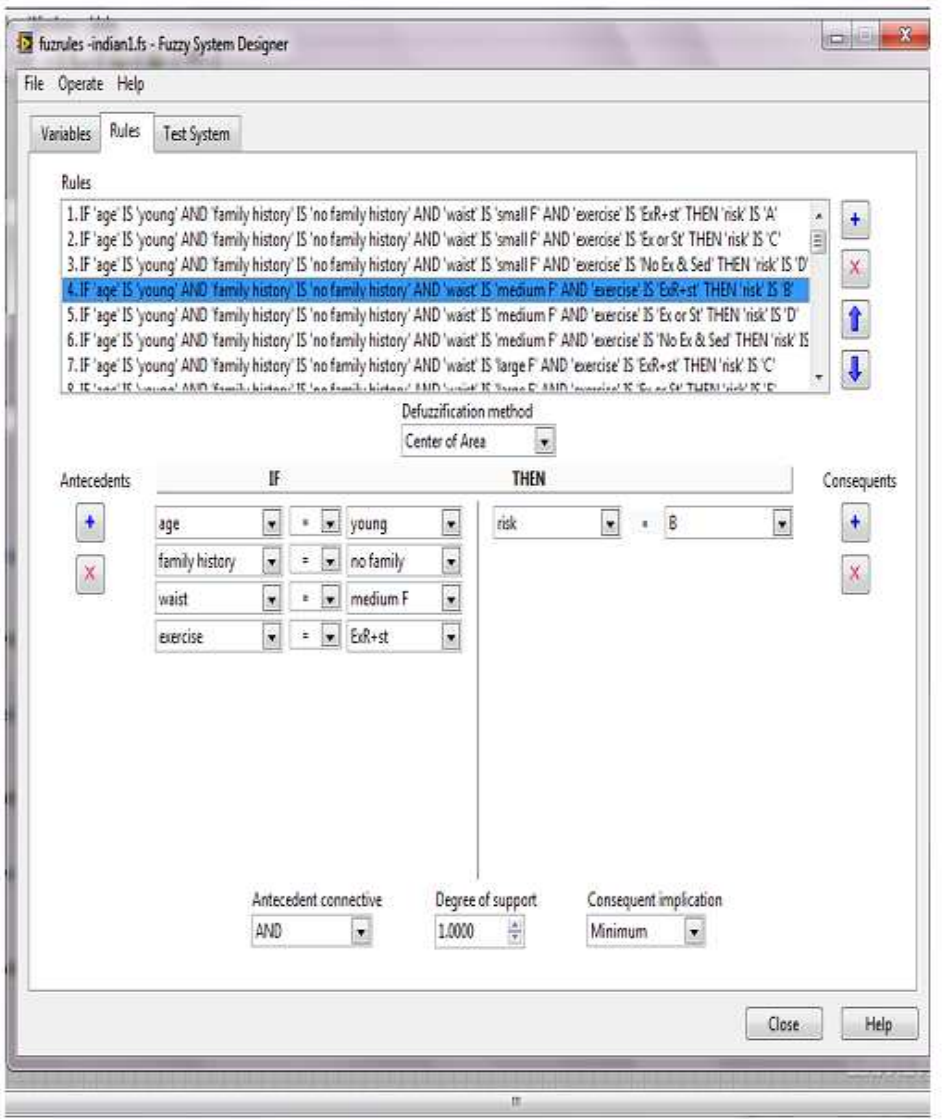

Fig. 6. Rules creation window 
Medical monitoring systems will provide excellent and timely treatment to those located in even the remotest places.

\section{INSTRUMENTATION}

An interactive system has been designed in Lab VIEW where the individual can enter his or her details to obtain their risk to diabetes mellitus. They have a choice of choosing one of three risk scores selected in the study. The risk scores obtained and the individual records can be sent the physician through online webpage. The risk scores interactive system is designed to coherently adapt all three risk scores after individual fuzzy system implementation. The risk scores will be displayed on the user screen. It is also possible for the physician located elsewhere to view the results. The physician can suggest improvements in diet and life style to decrease one's risk to diabetes mellitus. The screen is a two way input output system thus enabling efficient and quick patient physician interaction. This has been designed using web publishing tool in Lab VIEW. The patient can also post questions on the interactive text box and get answers from the physician. The block diagram and front panel of the VI code are shown in Fig. 7-9.

The real time implementation of the system would require computers with internet access at specific locations and nothing more. The Lab VIEW program can be converted to exe and run on any computer without the software installation procedure. The online web page is shown in Fig. 10.

As a study experiment we set up a counter where common people could access the online webpage and contact the physician. It was found to be fairly effective and it also pointed out that a better implementation would include voice controlled programs so as to answer questions rather than typing. This would eliminate the barrier of reaching illiterate people living in villages. Also once the program is able to function in local language it would make an almost perfect tool for diabetic detection and prediction.

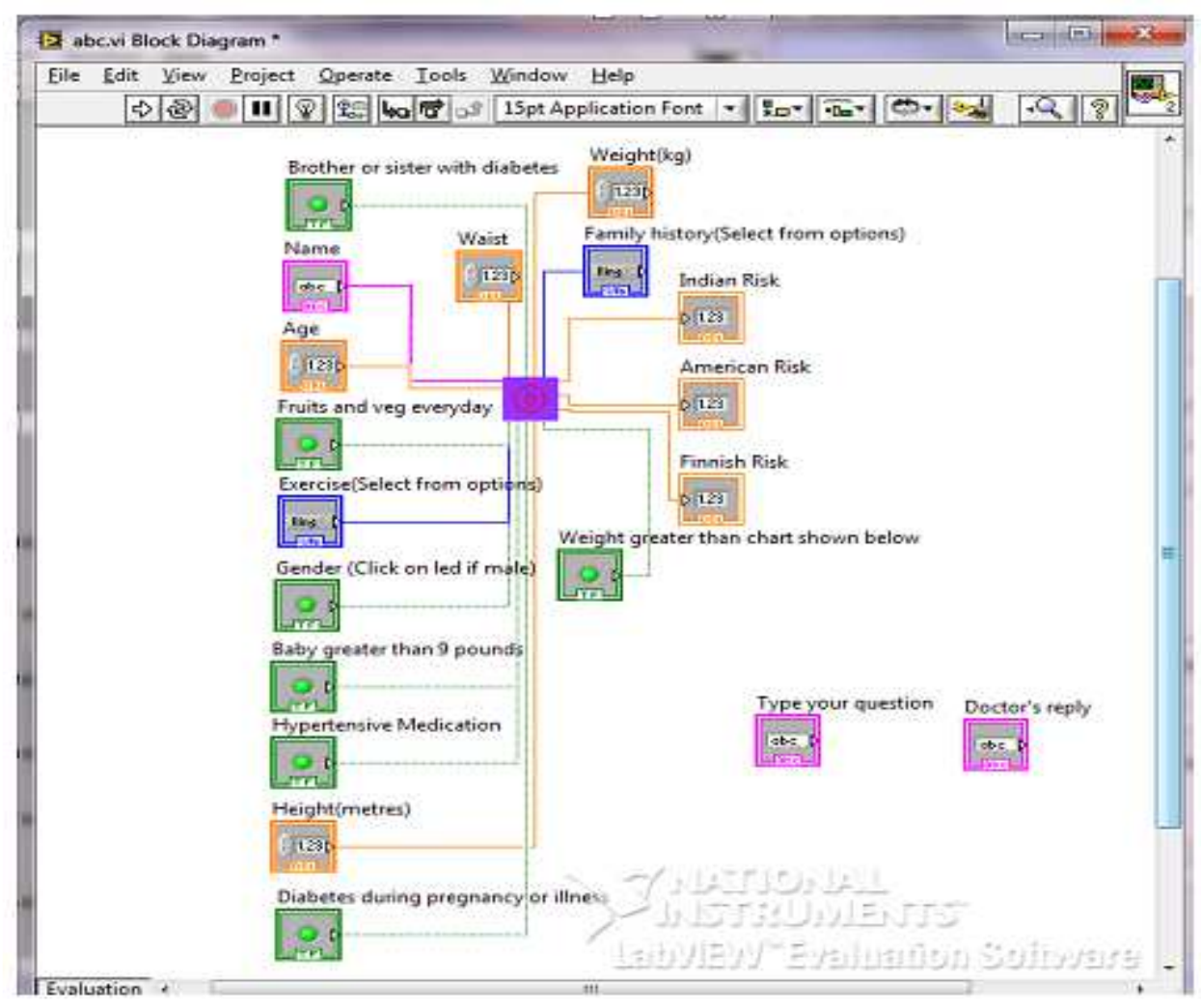

Fig. 7. Block Diagram of developed system 
SunithaKarnam Anantha et al. / American Journal of Applied Sciences 10 (9): 1124-1133, 2013

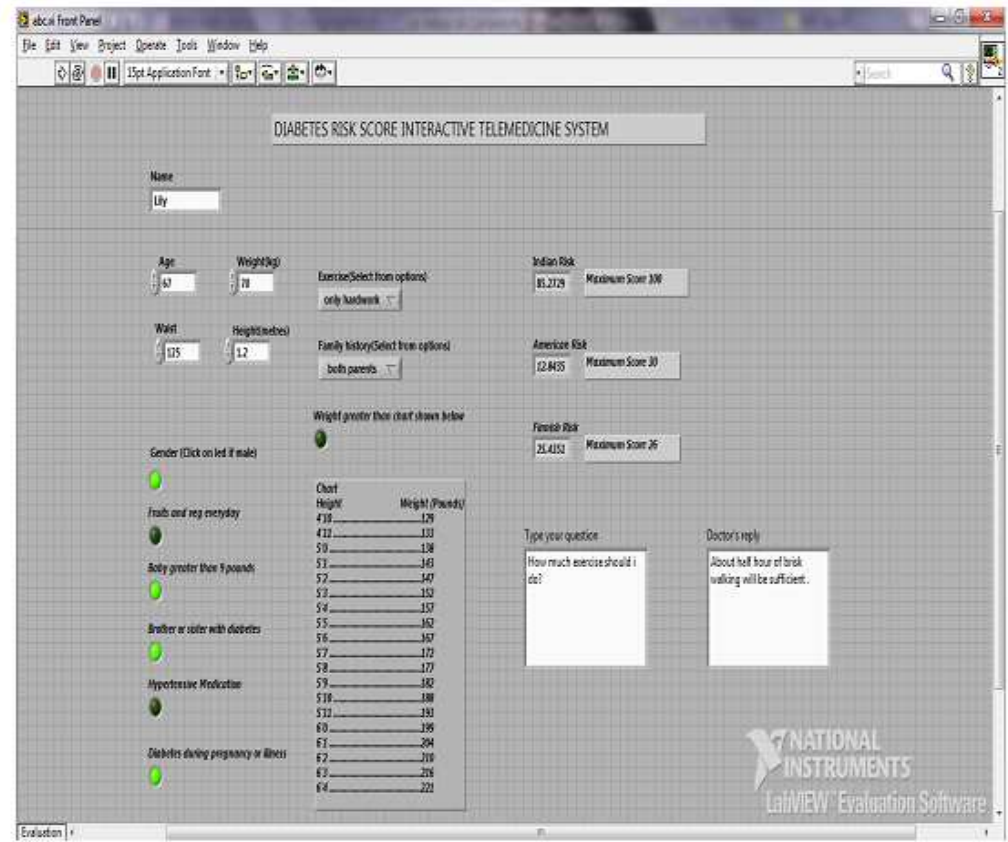

Fig. 8. Front panel of developed system

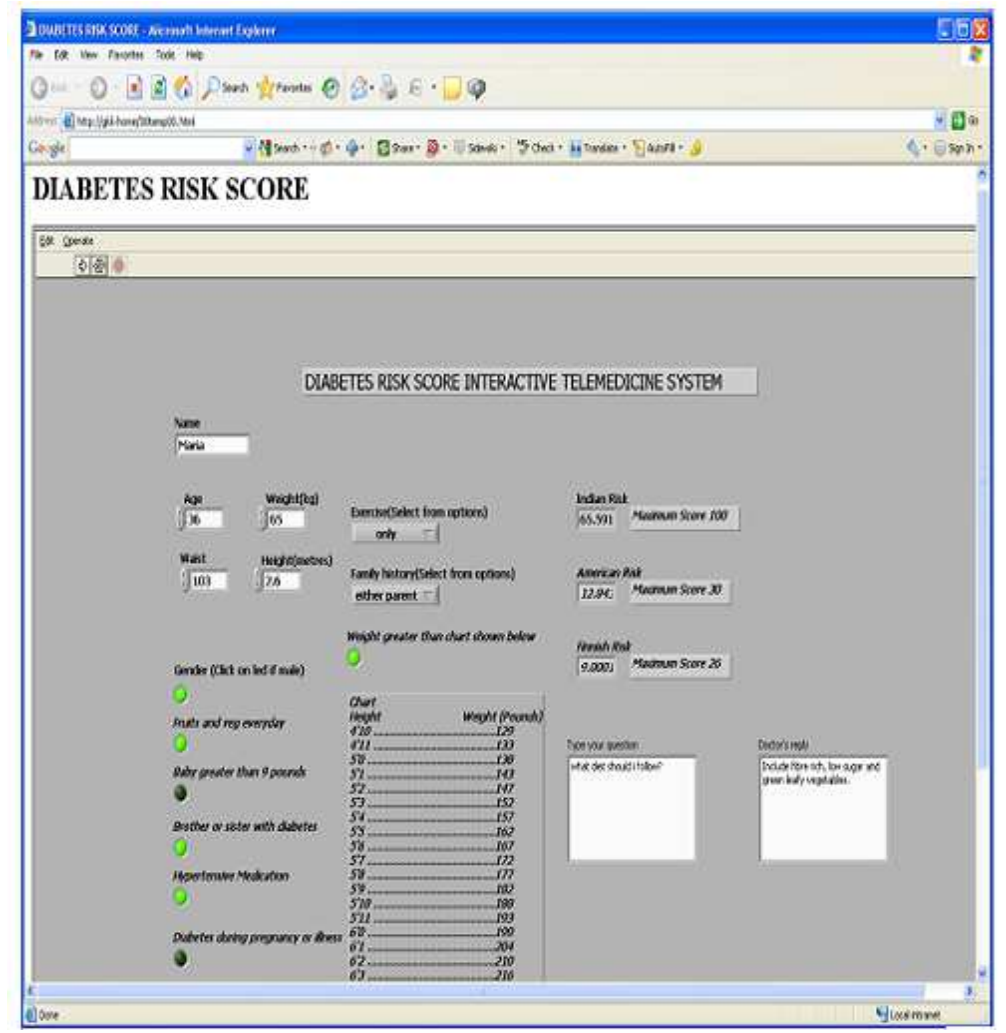

Fig. 9. Block diagram showing sub vi's 
SunithaKarnam Anantha et al. / American Journal of Applied Sciences 10 (9): 1124-1133, 2013

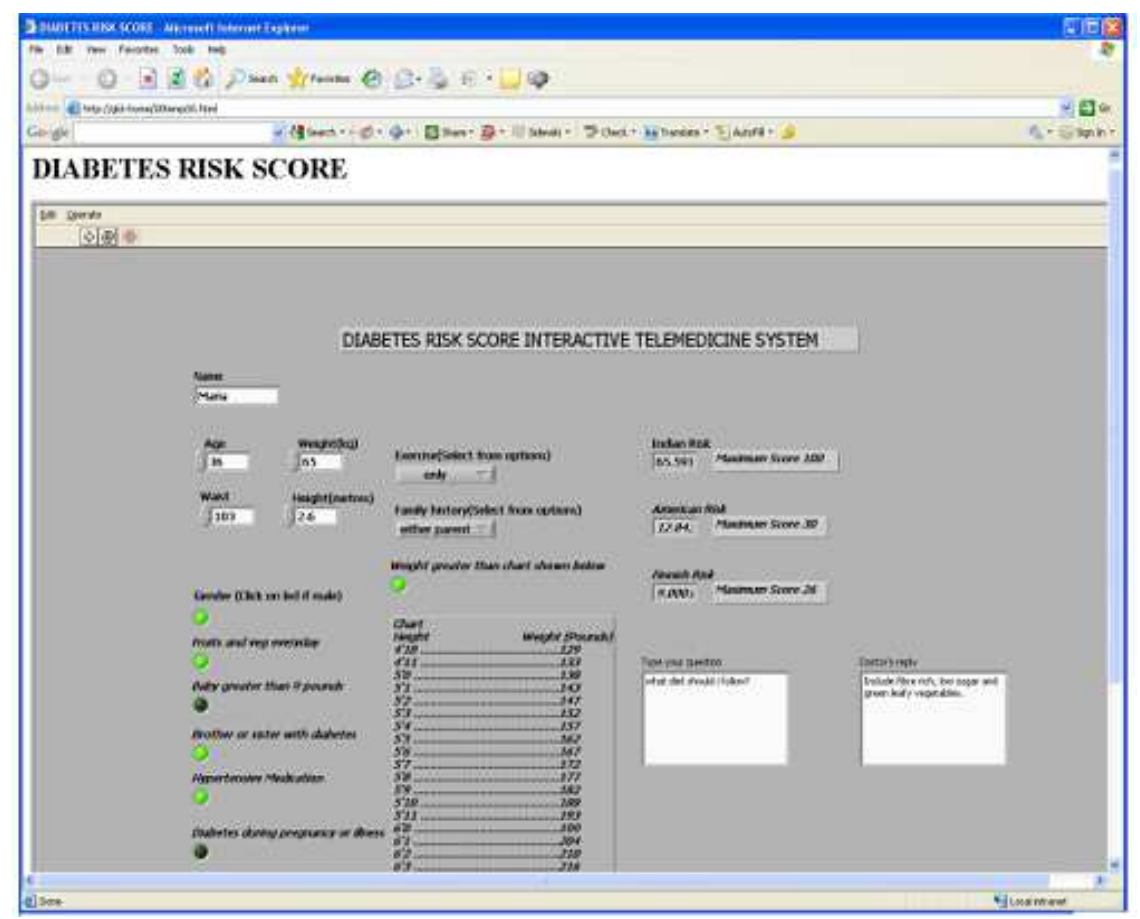

Fig. 10. Webpage display

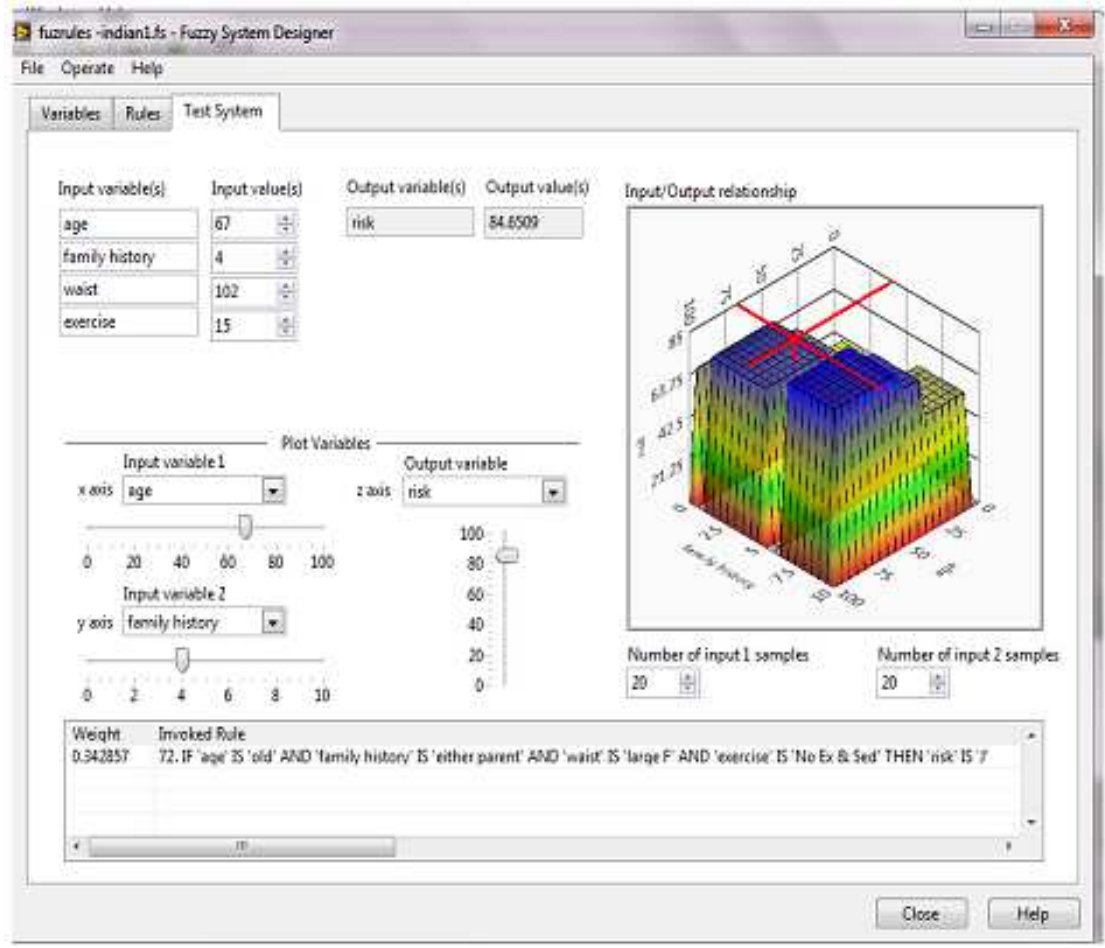

Fig. 11. Individual1 3D graph 


\section{OBSERVATION}

The data was obtained from a random population of individuals. The analysis was performed exclusively using IDRS because the individuals belong to Geographical location of southern India. The data was applied to normal and fuzzified systems to obtain the risk scores. Data was collected from about hundred Individuals. The fuzzy system was seen to provide more exacting results as compared to the normal system. A sample of 5 individuals from the collected Database is shown in Table 1.

The fuzzified data was found to be $5.9 \%$ approximately accurate than the normal system The distribution of the individual records according to the factors is shown in Table 2.
The 3D graphs were obtained for the above patient records. The graph shown in Fig. 11 shows risk score calculated for age of 67 , family history of either parent, Waist of 102 and moderate exercise. 3D Graphs can be plotted between any two input variables and the output variable. The graph shows the range of values each Variable can take. Here it has been plotted between age, family history and risk. The intersection of red lines shows the particular point obtained as a result of selected characters. One particular factor can be varied to observe the movement of point of intersection on the graph. Another instance with plot between age, Waist and risk is shown in Fig. 12.

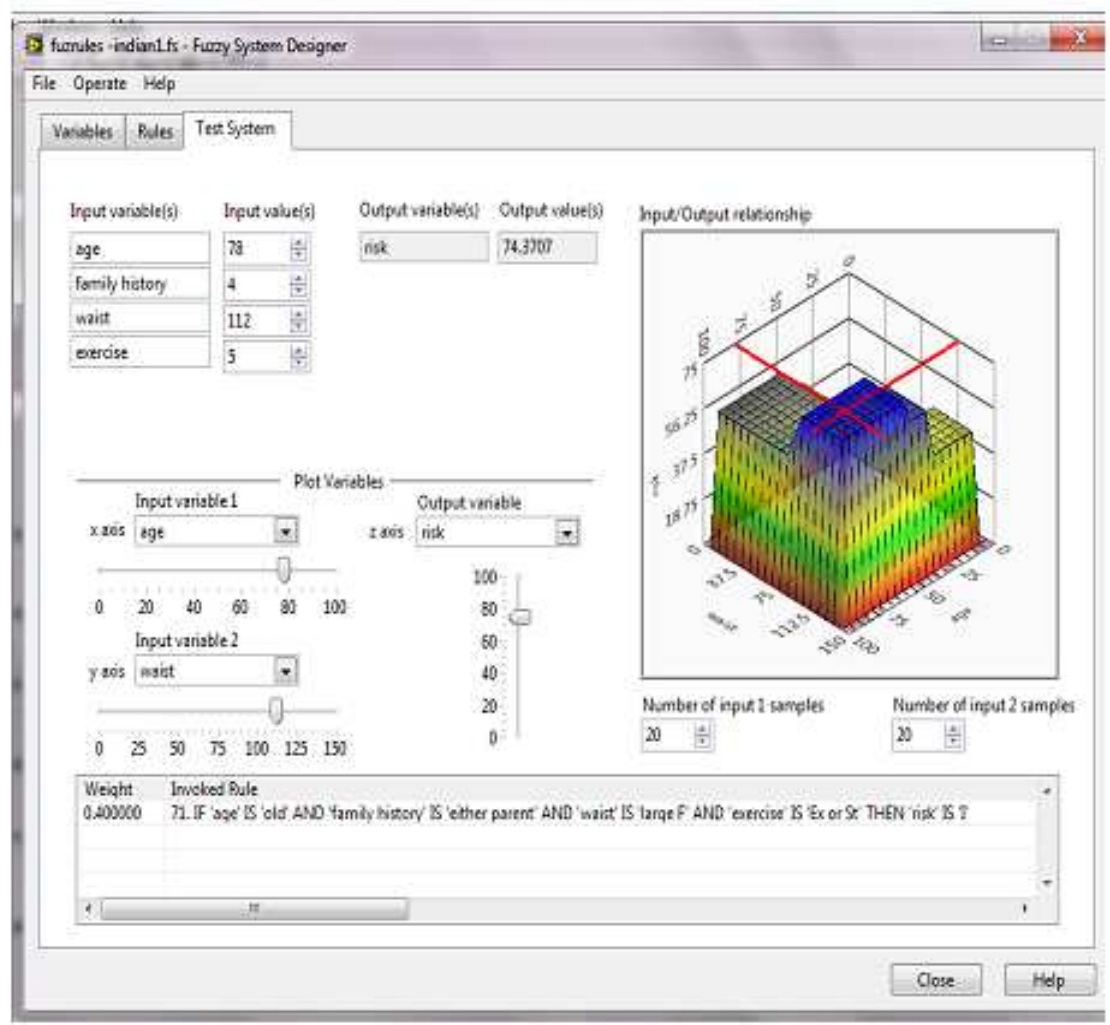

Fig. 12. Individual2 3D graph

Table 1. IDRS sample data

\begin{tabular}{lllllllr}
\hline Name & Age & Gender & Waist & Excersie & History & Idrs & Fuzzy \\
\hline A & 12 & Male & 61.0 & Medium & No & 20 & 15.46 \\
B & 23 & Male & 81.3 & High & Father & 10 & 4.76 \\
C & 50 & Female & 91.4 & Medium & Mother & 80 & 75.51 \\
D & 50 & Female & 81.3 & Medium & Mother & 70 & 65.31 \\
E & 60 & Female & 101.6 & Medium & Father & 80 & 75.39 \\
\hline
\end{tabular}


Table 2. Distribution of individual records

\begin{tabular}{llll}
\hline Factor & Category & Percentage & Total \\
\hline Gender & Male & 41 & 100 \\
& Female & 59 & 100 \\
Waist & HIGH & 16 & 73 \\
& MEDIUM & 11 & 100 \\
Excersie & LOW & 45 & 100 \\
& HIGH & 39 & 16 \\
Age & MEDIUM & 24 & \\
& LOW & 20 & 39 \\
& $0-20$ & 11 & 6 \\
\hline
\end{tabular}

\section{RESULTS}

The study is based upon an individual's records whose risk factors are given to each of the risk scores. The three different scores obtained are compared by taking a base score of hundred. The risk scores obtained directly from the table and fuzzified risk scores are compared with each other. The fuzzy system was found to be $5.9 \%$ accurate on the basis of the collected database. The fuzzified risk scores were found to be more accurate. The risk scores when sent to the physician via webpage helps him to analyze and improve one's lifestyle to reduce the risk scores. Lifestyle changes in terms of diet and daily exercise are extremely important for effective prevention and management of diabetes. The designed system will help to identify individuals at a high risk to diabetes at the earliest.

This will enable them to take precautions and delay diabetes to the maximum extent possible. This study can be expanded to more individuals and regions so as to obtain a comprehensive analysis of the risk to diabetes mellitus.

\section{CONCLUSION}

The fuzzy system was found to provide more accurate results as compared to normal system. The various patient record samples collected were used to obtain risk scores of normal and fuzzified IDRS. The results of the system are transmitted online through a webpage to obtain physician suggestions successfully.

\section{REFRENCES}

Aekplakorn, W., P. Bunnag and M. Woodward, 2006. A risk score for predicting incident diabetes in the Thai population. Diabetes Care, 29: 1872-1877. DOI: 10.2337/dc05-2141

Knowler, W.C., E. Barrett-Connor and S.E. Fowler, 2002. Reduction in the incidence of type 2 diabetes with lifestyle intervention or metformin. N Engl. J. Med., 346: 393-403. PMID: 11832527

Lindstrom, J. and J. Tuomilehto, 2003. The diabetes risk score: A practical tool to predict type 2 diabetes risk. Diabetes Care, 26: 725-731. PMID: 12610029

Mohan, V., M. Deepa, R. Deepa, S. Somannavar and M. Datta, 2005. Score for screening undiagnosed diabetes subjects-the Chennai Urban Rural. Epidemiol. Study (CURES-23), 53: 759-763.

Schulze, M.B., K. Hoffmann and H. Boeing, 2007. An accurate risk score based on anthropometric, dietary and lifestyle factors to predict the development of type 2 diabetes. Diabetes Care, 30: 510-51. PMID: 17327313

Wild, S., G. Roglic, A. Green, R. Sicree and H. King, 2004. Global prevalence of diabetes: Estimates for the year 2000 and projections for 2030. Diabetes Care, 27: 1047-1053. PMID: 15111519 